Stochastics (2007) 79(1-2) (139-154)

Discussion Paper No. 2006-058 of Sonderforschungsbereich 649 Economic Risk (22 pp)

\title{
Perpetual barrier options in jump-diffusion models*
}

\author{
Pavel V. Gapeev ${ }^{\dagger \ddagger}$
}

\begin{abstract}
We present a closed form solution to the perpetual American double barrier call option problem in a model driven by a Brownian motion and a compound Poisson process with exponential jumps. The method of proof is based on reducing the initial irregular optimal stopping problem to an integro-differential free-boundary problem and solving the latter by using continuous and smooth fit. The obtained solution of the nontrivial free-boundary problem gives the possibility to observe some special analytic properties of the value function at the optimal stopping boundaries.
\end{abstract}

Key words: American double barrier options, optimal stopping problem, jump-diffusion model, integro-differential free-boundary problem, continuous and smooth fit, Itô-Tanaka-Meyer formula. MSC (2000): Primary 60G40, 34K10, 91B28. Secondary 60J60, 60J75. JEL Classification: G13.

\section{Introduction}

The main aim of this paper is to present a closed form solution to the optimal stopping problem (2.3) for the process $S=\left(S_{t}\right)_{t \geq 0}$ defined in (2.1)-(2.2). This problem is related to the option pricing theory in mathematical finance, where the process $S$ can describe

*This research was supported by Deutsche Forschungsgemeinschaft through SFB 649 Economic Risk.

${ }^{\dagger}$ Weierstraß Institute for Applied Analysis and Stochastics (WIAS), Mohrenstr. 39, D-10117 Berlin, Germany, e-mail: gapeev@wias-berlin.de

${ }^{\ddagger}$ Institute of Control Sciences, Russian Academy of Sciences, Profsoyuznaya Str. 65, 117997 Moscow, Russia 
the price of a risky asset (e.g., a stock) on a financial market. In that case, the value (2.3) can be interpreted as a fair price of a perpetual double barrier call option of American type in a jump-diffusion model. The explicit expressions for the value function and the stopping boundary are derived by means of reducing the initial irregular optimal stopping problem (2.3) to the corresponding nontrivial integro-differential free-boundary problem (2.6)-(2.10) and solving the latter by applying continuous- and smooth-fit conditions. We note that the chosen approach based on reducing the initial optimal stopping problem to solving the associated free-boundary problem provides more valuable information on the nature of the solution than the standard so-called guess-and-verify approach. More precisely, this approach gives the possibility to see the difference of the structure of the solution under different relationships on the parameters of the model. Moreover, it can be observed explicitly that the value function (2.3) may not be smooth at the stopping boundary $B_{*}$ and may not be continuous at the point of discontinuity $L$ of the payoff function under some relationships on the parameters of the model. Such properties can be explained by the sample path behavior of the jump-diffusion process $S$ from (2.1)(2.2) as well as by the discontinuity of the reward in (2.3). The regularity of the value function for optimal stopping problems for Markov processes with discontinuous rewards and viscosity solutions of the related variational inequalities were studied in [2]-[3] and $[5]$.

For the classical Black-Merton-Scholes model driven by Brownian motion the problem (2.3) was considered in [4] for the single barrier case and both finite and infinite horizon, where the influence of the upper barrier on the stopping boundary was observed. The single upper barrier perpetual American put option problem with and without constraints on the short-selling of stock was considered in [13]. The closed-form expressions for the prices and optimal hedging strategies were obtained and the related stochastic optimization problem of mixed optimal stopping and singular control type in the constrained case was studied. The barrier version of the Russian option problem, where the decision about stopping should be taken before the price process reaches a 'dangerous' positive level, was recently studied in [22].

In the present paper we study a more general model by adding a compound Poisson 
process as driving term, where to simplify the exposition and aiming at closed form expressions for the value function and the stopping boundary we consider the perpetual case and let the jumps be exponentially distributed. Besides the analytical tractability of this model, it has some other desirable properties. For example, it is able to reproduce the leptokurtic feature of the return distribution. In addition, taking a HARA-type utility function and the corresponding utility-based martingale measure, the jumps remain exponentially distributed under the measure transformation (see [14]-[15] and also [16]-[17] for a detailed description of the model). Note that the obtained perpetual option prices can be considered as upper estimations for arbitrage-free prices of the related options with finite expiry which are widely used by practitioners. The barrier options of European type in more general exponential Lévy models were recently considered in [6], where the precise link between option prices and related partial integro-differential equations was explored.

The paper is organized as follows. In Section 2 we formulate the corresponding optimal stopping problem and reduce it to an equivalent integro-differential free-boundary problem. In Section 3 we derive an explicit solution to the free-boundary problem that also prepares the proof of the main result which is stated in Theorem 4.1. In Section 4 we verify that the solution of the free-boundary problem turns out to be a solution of the initial optimal stopping problem. In Section 5 we give some concluding remarks and comment the structure of the solution under different relationships on the parameters of the model.

\section{Formulation of the problem}

Let us now formulate the related irregular optimal stopping problem for a discontinuous reward and reduce it to the equivalent free-boundary problem.

2.1. For a precise formulation of the problem let us consider a probability space $(\Omega, \mathcal{F}, P)$ with a standard Brownian motion $W=\left(W_{t}\right)_{t \geq 0}$ and a jump process $J=\left(J_{t}\right)_{t \geq 0}$ defined by $J_{t}=\sum_{i=1}^{N_{t}} Y_{i}$, where $N=\left(N_{t}\right)_{t \geq 0}$ is a Poisson process with intensity $\lambda$ and $\left(Y_{i}\right)_{i \in \mathbb{N}}$ is a sequence of independent random variables exponentially distributed with parameter $1\left(W, N\right.$ and $\left(Y_{i}\right)_{i \in \mathbb{N}}$ are supposed to be independent). The stock price process 
$S=\left(S_{t}\right)_{t \geq 0}$ is given by:

$$
S_{t}=s \exp \left(\left(r-\delta-\frac{\sigma^{2}}{2}-\frac{\lambda \theta}{1-\theta}\right) t+\sigma W_{t}+\theta J_{t}\right)
$$

where $\sigma \geq 0,0 \leq \delta<r$ and $\theta<1$. It follows that $S$ solves the stochastic differential equation:

$$
d S_{t}=S_{t-}(r-\delta) d t+S_{t-} \sigma d W_{t}+S_{t-} \int_{0}^{\infty}\left(e^{\theta y}-1\right)(\mu(d t, d y)-\nu(d t, d y)) \quad\left(S_{0}=s\right)
$$

where $r$ is the riskless interest rate and the dividend rate payed to stockholders is $\delta S_{t}$. Here $\mu(d t, d y)$ is the measure of jumps of the process $J$ with the compensator $\nu(d t, d y)=$ $\lambda d t I(y>0) e^{-y} d y$, which means that we work directly under a martingale measure for $S$. Note that the assumption $\theta<1$ guarantees that the jumps of $S$ are integrable under the martingale measure, which is no restriction.

By using the utility arguments presented in the previous section (see also [12] or [8]) we may conclude that an arbitrage-free price for the perpetual American double barrier call option coincides with the value of the following optimal stopping problem:

$$
V_{*}(s)=\sup _{\tau} E_{s}\left[e^{-r \tau}\left(S_{\tau}-K\right)^{+} I(\tau<\eta)\right]
$$

where the supremum is taken over all stopping times $\tau$ with respect to the natural filtration of $S$, and $E_{s}$ denotes the expectation under the assumption that $S_{0}=s$ for $s>0$. Here we set $\eta=\inf \left\{t \geq 0 \mid S_{t} \notin(L, H)\right\}$ for some $0<L<K<H$ given and fixed, and observe that $V_{*}(s)=0$ for all $0<s \leq L$ and $s \geq H$. We also note that when $\delta=0$ the solution of the problem (2.3) can be trivial (under $H \uparrow \infty$ ), so that we assume that $\delta>0$. Taking into account the structure of the payoff function in the problem (2.3), we will search for an optimal stopping time in the form:

$$
\tau_{*}=\inf \left\{t \geq 0 \mid S_{t} \notin\left(L, B_{*}\right)\right\}
$$

for some number $B_{*} \in(K, H)$ to be determined. The structure of (2.4) can be explained by the fact that as in the case of standard perpetual American call option the process $S$ should be stopped after it overlaps the upper boundary $B_{*}$, but this should be done before it passes through one of the barriers $L$ or $H$. Note that, so far the process $S$ has 
not passed through the lower barrier $L$, there should be a possibility for it to reach the exercise boundary $B_{*} \in(K, H)$ when the latter exists.

2.2. By means of standard arguments it can be shown that the infinitesimal operator $\mathbb{L}$ of the process $S$ acts on an arbitrary bounded function $F \in C_{b}^{2}(0, \infty)\left(\right.$ or $F \in C_{b}^{1}(0, \infty)$ when $\sigma=0$ ) according to the rule:

$$
(\mathbb{L} F)(s)=(r-\delta+\zeta) s F^{\prime}(s)+\frac{\sigma^{2}}{2} s^{2} F^{\prime \prime}(s)+\int_{0}^{\infty}\left(F\left(s e^{\theta y}\right)-F(s)\right) \lambda e^{-y} d y
$$

for all $s>0$, where we denote $\zeta=-\lambda \theta /(1-\theta)$. In order to find explicit expressions for the unknown value function $V_{*}(s)$ from (2.3) and the unknown boundary $B_{*}$ from (2.4), let us use the results of general theory of optimal stopping problems for continuous time Markov processes (see, e.g., [9], [24, Chapter III, Section 8] and [20]). We can reduce the optimal stopping problem (2.3) to the free-boundary problem:

$$
\begin{aligned}
& (\mathbb{L} V)(s)=r V(s) \quad \text { for } \quad L<s<B \\
& V(B-)=B-K, \quad V(L+)=0 \text { if either } \sigma>0 \text { or } r-\delta+\zeta<0 \\
& V(s)=s-K \text { for } B \leq s<H, \quad V(s)=0 \text { for } 0<s \leq L \text { and } s \geq H \\
& V(s) \geq(s-K)^{+} \text {for } L<s<B
\end{aligned}
$$

for some $0<K<B<H$. The first equality in (2.7) is the instantaneous-stopping condition playing the role of the continuous-fit condition in case $\sigma=0$, and the second equality in (2.7) is the continuity condition for the value function at the fixed point $L$, which is a discontinuity point for the payoff function. The similar properties were observed in [6] by solving barrier option problems of another European type with fixed time expiry and discontinuous payoffs in models with jumps. Note that the superharmonic characterization of the value function (see [7], [24] and [20]) implies that (2.3) is the smallest function satisfying (2.6)-(2.9). Moreover, we further assume that the smooth-fit condition:

$$
V^{\prime}(B-)=1 \text { if either } \sigma>0 \text { or } r-\delta+\zeta>0
$$

is satisfied for $0<K<B<H$. The latter can be explained by the fact that in those cases, leaving the continuation region $\left(L, B_{*}\right)$ the process $S$ can pass through the boundary 
$B_{*}<H$ continuously. This property was earlier observed and explained in [18, Section 2] and [19] by solving some other optimal stopping problems for jump processes (see also [1] for necessary and sufficient conditions for the occurrence of smooth fit and references to the related literature, and [20] for an extensive overview).

\section{Solution of the free-boundary problem}

Let us now derive explicit solutions to the free-boundary problem formulated above under different relationships on the parameters of the model.

3.1. Let us first consider the continuous case $\sigma>0$ and $\theta=0$. In this case, by means of the same arguments as in [23, Section 8] or [25, Chapter VII, Section 2a], it can be shown that the equation (2.6) has the general solution:

$$
V(s)=C_{1} s^{\gamma_{1}}+C_{2} s^{\gamma_{2}}
$$

where $C_{1}$ and $C_{2}$ are some arbitrary constants, and $\gamma_{2}<0<1<\gamma_{1}$ are given by:

$$
\gamma_{i}=\frac{1}{2}-\frac{r-\delta}{\sigma^{2}}-(-1)^{i} \sqrt{\left(\frac{1}{2}-\frac{r-\delta}{\sigma^{2}}\right)^{2}+\frac{2 r}{\sigma^{2}}}
$$

for $i=1,2$. Hence, applying the conditions (2.7) and (2.10) to the function (3.1), we get that the following equalities:

$$
\begin{aligned}
& C_{1} B^{\gamma_{1}}+C_{2} B^{\gamma_{2}}=B-K \\
& C_{1} L^{\gamma_{1}}+C_{2} L^{\gamma_{2}}=0 \\
& \gamma_{1} C_{1} B^{\gamma_{1}}+\gamma_{2} C_{2} B^{\gamma_{2}}=B
\end{aligned}
$$

hold for some $0<K<B<H$. Thus, solving the system (3.3)-(3.5) we get that the solution of the problem $(2.6)-(2.7)+(2.10)$ is given by:

$$
V\left(s ; B_{*}\right)=\left(B_{*}-K\right) \frac{(s / L)^{\gamma_{1}}-(s / L)^{\gamma_{2}}}{\left(B_{*} / L\right)^{\gamma_{1}}-\left(B_{*} / L\right)^{\gamma_{2}}}
$$

for all $0<L<s<B_{*}$, where $B_{*}$ is determined as the unique solution of the equation:

$$
\frac{\gamma_{1}(B / L)^{\gamma_{1}}-\gamma_{2}(B / L)^{\gamma_{2}}}{(B / L)^{\gamma_{1}}-(B / L)^{\gamma_{2}}}=\frac{B}{B-K}
$$


whenever its unique root belongs to the interval $(K, H)$. The uniqueness of the root of (3.7) on the interval $(K, \infty)$ for each $L \in(0, K)$ fixed is easier to recognize after we rewrite the left- and right-hand sides as $\gamma_{1}+\left(\gamma_{1}-\gamma_{2}\right) /\left[(B / L)^{\gamma_{1}-\gamma_{2}}-1\right]$ and $1+1 /(B / K-1)$, respectively, and recall that $\gamma_{2}<0<1<\gamma_{1}$.

Observe that when $L=0$, taking into account the fact that $\gamma_{2}<0<1<\gamma_{1}$, it follows that in (3.1) we have $C_{2}=0$, since otherwise $V(s) \rightarrow \pm \infty$ as $s \downarrow 0$, which should be excluded by virtue of the obvious fact that the value function (2.3) is bounded under $s \downarrow 0$. Note that the same conclusion can be made based on the argument that 0 is a natural boundary for the process $S$ in this case. Thus, solving the system $(3.3)+(3.5)$ with $C_{2}=0$, we get that the solution of the problem (2.6)-(2.7) $+(2.10)$ takes the form:

$$
V\left(s ; B_{*}\right)=\left(B_{*}-K\right)\left(\frac{s}{B_{*}}\right)^{\gamma_{1}}
$$

for all $L<s<B_{*}$, where $B_{*}$ is given by:

$$
B_{*}=\frac{\gamma_{1} K}{\gamma_{1}-1}
$$

whenever $B_{*} \in(K, H)$. The formulas (3.8) and (3.9) were earlier obtained in [4, Section 1].

3.2. From now on let us consider the jump-diffusion case $\theta \neq 0$ and for the integrability of jumps assume that $\theta<1$. By means of straightforward calculations, we reduce the equation (2.6) to the form:

$$
(r-\delta+\zeta) s V^{\prime}(s)+\frac{\sigma^{2}}{2} s^{2} V^{\prime \prime}(s)-\alpha \lambda s^{\alpha} G(s)=(r+\lambda) V(s)
$$

with $\alpha=1 / \theta$ and $\zeta=-\lambda \theta /(1-\theta)$, where taking into account the conditions $(2.7)-(2.8)$ we set:

$$
\begin{aligned}
& G(s)=-\int_{s}^{B} V(z) \frac{d z}{z^{\alpha+1}}+F(B, H, K) \quad \text { if } \quad \alpha=1 / \theta>1 \\
& G(s)=\int_{L}^{s} V(z) \frac{d z}{z^{\alpha+1}} \quad \text { if } \quad \alpha=1 / \theta<0
\end{aligned}
$$

for all $0<L<s \leq B$ and denote:

$$
F(B, H, K)=\frac{\alpha B+(1-\alpha) K}{B^{\alpha} \alpha(1-\alpha)}-\frac{\alpha H+(1-\alpha) K}{H^{\alpha} \alpha(1-\alpha)}
$$


for each $0<K<B<H$. Then, from (3.10) and (3.11)-(3.12) it follows that the function $G(s)$ solves the following (third-order) ordinary differential equation:

$$
\begin{aligned}
& \frac{\sigma^{2} s^{3}}{2} G^{\prime \prime \prime}(s)+\left[\sigma^{2}(\alpha+1)+r-\delta+\zeta\right] s^{2} G^{\prime \prime}(s) \\
& +\left[(\alpha+1)\left(\frac{\sigma^{2} \alpha}{2}+r-\delta+\zeta\right)-(r+\lambda)\right] s G^{\prime}(s)-\alpha \lambda G(s)=0
\end{aligned}
$$

for $0<L<s<B$, which has the general solution:

$$
G(s)=C_{1} \frac{s^{\beta_{1}}}{\beta_{1}}+C_{2} \frac{s^{\beta_{2}}}{\beta_{2}}+C_{3} \frac{s^{\beta_{3}}}{\beta_{3}}
$$

where $C_{1}, C_{2}$ and $C_{3}$ are some arbitrary constants and $\beta_{3}<\beta_{2}<\beta_{1}, \beta_{i} \neq 0$ for $i=1,2,3$, are the real roots of the corresponding (characteristic) equation:

$$
\begin{aligned}
& \frac{\sigma^{2}}{2} \beta^{3}+\left[\sigma^{2}\left(\alpha-\frac{1}{2}\right)+r-\delta+\zeta\right] \beta^{2} \\
& +\left[\alpha\left(\frac{\sigma^{2}(\alpha-1)}{2}+r-\delta+\zeta\right)-(r+\lambda)\right] \beta-\alpha \lambda=0 .
\end{aligned}
$$

Therefore, differentiating both sides of the formulas (3.11)-(3.12) we obtain that the integro-differential equation (3.10) has the general solution:

$$
V(s)=C_{1} s^{\gamma_{1}}+C_{2} s^{\gamma_{2}}+C_{3} s^{\gamma_{3}}
$$

where we set $\gamma_{i}=\beta_{i}+\alpha$ for $i=1,2,3$. Observe that if $\sigma=0$ and $r-\delta+\zeta \neq 0$ then it is seen that (3.14) degenerates into a second-order differential equation, and in that case we can put $C_{3}=0$ into (3.15) and (3.17), while the roots of the equation (3.16) are explicitly given by:

$$
\beta_{i}=\frac{r+\lambda}{2(r-\delta+\zeta)}-\frac{\alpha}{2}-(-1)^{i} \sqrt{\left(\frac{r+\lambda}{2(r-\delta+\zeta)}-\frac{\alpha}{2}\right)^{2}+\frac{\alpha \lambda}{r-\delta+\zeta}}
$$

for $i=1,2$. Note that if $\sigma=0$ and $r-\delta+\zeta=0$ then (3.14) degenerates into a first-order differential equation, and in that case we can put $C_{2}=C_{3}=0$ into (3.15) and (3.17), while the unique root of the equation (3.16) is given by:

$$
\beta_{1}=-\frac{\alpha \lambda}{r+\lambda}
$$


Hence, by applying conditions (3.11)-(3.12), (2.7) and (2.10) to the functions (3.15) and (3.17), respectively, we obtain that the following equalities:

$$
\begin{aligned}
& C_{1} \frac{B^{\gamma_{1}}}{\beta_{1}}+C_{2} \frac{B^{\gamma_{2}}}{\beta_{2}}+C_{3} \frac{B^{\gamma_{3}}}{\beta_{3}}=B^{\alpha} F(B, H, K) \\
& C_{1} \frac{L^{\gamma_{1}}}{\beta_{1}}+C_{2} \frac{L^{\gamma_{2}}}{\beta_{2}}+C_{3} \frac{L^{\gamma_{3}}}{\beta_{3}}=0 \\
& C_{1} B^{\gamma_{1}}+C_{2} B^{\gamma_{2}}+C_{3} B^{\gamma_{3}}=B-K \\
& C_{1} L^{\gamma_{1}}+C_{2} L^{\gamma_{2}}+C_{3} L^{\gamma_{3}}=0 \\
& \gamma_{1} C_{1} B^{\gamma_{1}}+\gamma_{2} C_{2} B^{\gamma_{2}}+\gamma_{3} C_{3} B^{\gamma_{3}}=B
\end{aligned}
$$

hold for some $0<K<B<H$ with $F(B, H, K)$ defined in (3.13). Here (3.20) holds if $0<\theta<1,(3.21)$ holds if $\theta<0$, (3.23) holds if either $\sigma>0$ or $r-\delta+\zeta<0$ with $\zeta=-\lambda \theta /(1-\theta)$, and (3.24) holds if either $\sigma>0$ or $r-\delta+\zeta>0$. Below we determine the unknown constants $C_{i}$ for $i=1,2,3$ and the optimal boundary $B_{*}$ under different relationships on the parameters of the model.

3.3. Let us now consider the subcase of negative jumps $\alpha=1 / \theta<0$. If, in addition, $\sigma>0$ holds, then solving the system (3.21)-(3.24), by using straightforward calculations we obtain that the solution of the system $(2.6)-(2.8)+(2.10)$ is given by:

$$
V\left(s ; B_{*}\right)=\left(B_{*}-K\right) \frac{\beta_{1}\left(\beta_{3}-\beta_{2}\right)(s / L)^{\gamma_{1}}+\beta_{2}\left(\beta_{1}-\beta_{3}\right)(s / L)^{\gamma_{2}}+\beta_{3}\left(\beta_{2}-\beta_{1}\right)(s / L)^{\gamma_{3}}}{\beta_{1}\left(\beta_{3}-\beta_{2}\right)\left(B_{*} / L\right)^{\gamma_{1}}+\beta_{2}\left(\beta_{1}-\beta_{3}\right)\left(B_{*} / L\right)^{\gamma_{2}}+\beta_{3}\left(\beta_{2}-\beta_{1}\right)\left(B_{*} / L\right)^{\gamma_{3}}}
$$

for all $0<L<s<B_{*}$, where $B_{*}$ is determined as the unique solution of the equation:

$$
\frac{\beta_{1}\left(\beta_{3}-\beta_{2}\right) \gamma_{1}(B / L)^{\gamma_{1}}+\beta_{2}\left(\beta_{1}-\beta_{3}\right) \gamma_{2}(B / L)^{\gamma_{2}}+\beta_{3}\left(\beta_{2}-\beta_{1}\right) \gamma_{3}(B / L)^{\gamma_{3}}}{\beta_{1}\left(\beta_{3}-\beta_{2}\right)(B / L)^{\gamma_{1}}+\beta_{2}\left(\beta_{1}-\beta_{3}\right)(B / L)^{\gamma_{2}}+\beta_{3}\left(\beta_{2}-\beta_{1}\right)(B / L)^{\gamma_{3}}}=\frac{B}{B-K}
$$

whenever its unique root belongs to the interval $(K, H)$. The uniqueness of the root of (3.26) on the interval $(K, \infty)$ for each $L \in(0, K)$ fixed is easier to recognize after we rewrite the left- and right-hand sides as $\gamma_{1}+\left[\beta_{2}\left(\beta_{1}-\beta_{3}\right)\left(\gamma_{2}-\gamma_{1}\right)(B / L)^{\gamma_{2}}+\beta_{3}\left(\beta_{2}-\right.\right.$ $\left.\left.\beta_{1}\right)\left(\gamma_{3}-\gamma_{1}\right)(B / L)^{\gamma_{3}}\right] /\left[\beta_{1}\left(\beta_{3}-\beta_{2}\right)(B / L)^{\gamma_{1}}+\beta_{2}\left(\beta_{1}-\beta_{3}\right)(B / L)^{\gamma_{2}}+\beta_{3}\left(\beta_{2}-\beta_{1}\right)(B / L)^{\gamma_{3}}\right]$ and $1+1 /(B / K-1)$, respectively, and notice that if $\alpha=1 / \theta<0$ then $\beta_{3}<0<\beta_{2}<-\alpha<$ $1-\alpha<\beta_{1}$ so that $\gamma_{3}<\alpha<\gamma_{2}<0<1<\gamma_{1}$ with $\gamma_{i}=\beta_{i}+\alpha$ for $i=1,2,3$.

Note that if, in addition, $\sigma=0$ holds, then we can put $C_{3}=0$ into (3.15) and (3.17) and omit the second condition in (2.7) implying (3.23). Thus, solving the system 
(3.21)-(3.22) $+(3.24)$ with $C_{3}=0$, by using straightforward calculations we obtain that the solution of the system $(2.6)-(2.8)+(2.10)$ is given by:

$$
V\left(s ; B_{*}\right)=\left(B_{*}-K\right) \frac{\beta_{1}(s / L)^{\gamma_{1}}-\beta_{2}(s / L)^{\gamma_{2}}}{\beta_{1}\left(B_{*} / L\right)^{\gamma_{1}}-\beta_{2}\left(B_{*} / L\right)^{\gamma_{2}}}
$$

for all $0<L<s<B_{*}$, where $B_{*}$ is determined as the unique solution of the equation:

$$
\frac{\beta_{1} \gamma_{1}(B / L)^{\gamma_{1}}-\beta_{2} \gamma_{2}(B / L)^{\gamma_{2}}}{\beta_{1}(B / L)^{\gamma_{1}}-\beta_{2}(B / L)^{\gamma_{2}}}=\frac{B}{B-K}
$$

whenever its unique root belongs to the interval $(K, H)$. The uniqueness of the root of $(3.28)$ on the interval $(K, \infty)$ for each $L \in(0, K)$ fixed is easier to recognize after we rewrite the left- and right-hand sides as $\gamma_{1}+\left(\gamma_{1}-\gamma_{2}\right) /\left[\left(\beta_{1} / \beta_{2}\right)(B / L)^{\gamma_{1}-\gamma_{2}}-1\right]$ and $1+1 /(B / K-1)$, respectively.

Observe that when $L=0$ we omit the second condition in (2.7) implying (3.23) as well as (3.21) and take into account the fact that if $\alpha=1 / \theta<0$ then $\beta_{3}<0<\beta_{2}<$ $-\alpha<1-\alpha<\beta_{1}$ so that $\gamma_{3}<\alpha<\gamma_{2}<0<1<\gamma_{1}$ with $\gamma_{i}=\beta_{i}+\alpha$ for $i=1,2,3$. It thus follows that in (3.15) as well as in (3.17) we have $C_{2}=C_{3}=0$, since otherwise $G(s) \rightarrow \pm \infty$ and $V(s) \rightarrow \pm \infty$ as $s \downarrow 0$ that should be excluded by virtue of the facts that the value function (2.3) so that the function (3.12) are bounded under $s \downarrow 0$. Therefore, solving the system $(3.22)+(3.24)$ with $C_{2}=C_{3}=0$ we obtain that the solution of the system $(2.6)-(2.8)+(2.10)$ is given by the same formulas as in (3.8)-(3.9) with $\gamma_{1}$ replaced by $\beta_{1}+\alpha$, where if $\sigma>0$ then $\beta_{1}$ is the largest root of the equation (3.16), and if $\sigma=0$ then $\beta_{1}$ is given by (3.18).

3.4. Let us now consider the subcase of positive jumps $\alpha=1 / \theta>1$. If, in addition, $\sigma>0$ holds, then solving the system $(3.20)+(3.22)-(3.24)$, by using straightforward calculations we obtain that the solution of the system $(2.6)-(2.8)+(2.10)$ is given by $(3.17)$ 
with $C_{i}=C_{i}\left(B_{*}, H, K, L\right)$ for $i=1,2,3$ defined by:

$$
\begin{aligned}
& C_{1}=\frac{\left[A\left(B_{*}, H, K\right)-\beta_{1} \beta_{3}\left(B_{*}-K\right)\right]\left(L^{\gamma_{2}} B_{*}^{\gamma_{3}}-L^{\gamma_{3}} B_{*}^{\gamma_{2}}\right)+\beta_{1}\left(B_{*}-K\right)\left(\beta_{3} L^{\gamma_{3}} B_{*}^{\gamma_{2}}-\beta_{2} L^{\gamma_{2}} B_{*}^{\gamma_{3}}\right)}{\beta_{3}\left(\beta_{2}-\beta_{1}\right) B_{*}^{\gamma_{1}}\left(L^{\gamma_{2}} B_{*}^{\gamma_{3}}-L^{\gamma_{3}} B_{*}^{\gamma_{2}}\right)-\beta_{1}\left(\beta_{2}-\beta_{3}\right) B_{*}^{\gamma_{3}}\left(L^{\gamma_{2}} B_{*}^{\gamma_{1}}-L^{\gamma_{1}} B_{*}^{\gamma_{2}}\right)} \\
& C_{2}=\frac{\left[A\left(B_{*}, H, K\right)-\beta_{1} \beta_{2}\left(B_{*}-K\right)\right]\left(L^{\gamma_{3}} B_{*}^{\gamma_{1}}-L^{\gamma_{1}} B_{*}^{\gamma_{3}}\right)+\beta_{2}\left(B_{*}-K\right)\left(\beta_{1} L^{\gamma_{1}} B_{*}^{\gamma_{3}}-\beta_{3} L^{\gamma_{3}} B_{*}^{\gamma_{1}}\right)}{\beta_{1}\left(\beta_{3}-\beta_{2}\right) B_{*}^{\gamma_{2}}\left(L^{\gamma_{3}} B_{*}^{\gamma_{1}}-L^{\gamma_{1}} B_{*}^{\gamma_{3}}\right)-\beta_{2}\left(\beta_{3}-\beta_{1}\right) B_{*}^{\gamma_{1}}\left(L^{\gamma_{3}} B_{*}^{\gamma_{2}}-L^{\gamma_{2}} B_{*}^{\gamma_{3}}\right)} \\
& C_{3}=\frac{\left[A\left(B_{*}, H, K\right)-\beta_{2} \beta_{3}\left(B_{*}-K\right)\right]\left(L^{\gamma_{1}} B_{*}^{\gamma_{2}}-L^{\gamma_{2}} B_{*}^{\gamma_{1}}\right)+\beta_{3}\left(B_{*}-K\right)\left(\beta_{2} L^{\gamma_{2}} B_{*}^{\gamma_{1}}-\beta_{1} L^{\gamma_{1}} B_{*}^{\gamma_{2}}\right)}{\beta_{2}\left(\beta_{1}-\beta_{3}\right) B_{*}^{\gamma_{3}}\left(L^{\gamma_{1}} B_{*}^{\gamma_{2}}-L^{\gamma_{2}} B_{*}^{\gamma_{1}}\right)-\beta_{3}\left(\beta_{1}-\beta_{2}\right) B_{*}^{\gamma_{2}}\left(L^{\gamma_{1}} B_{*}^{\gamma_{3}}-L^{\gamma_{3}} B_{*}^{\gamma_{1}}\right)}
\end{aligned}
$$

for all $0<L<s<B_{*}$ with $A(B, H, K)=\beta_{1} \beta_{2} \beta_{3} B^{\alpha} F(B, H, K)$ for each $0<K<$ $B<H$, where $B_{*}$ is determined as the unique solution of the equation (3.24) with $C_{i}=C_{i}\left(B_{*}, H, K, L\right)$ for $i=1,2,3$ given by (3.29)-(3.31) on the interval $(K, H)$ whenever it exists.

Since in this subcase as well as in the other subcases below it is difficult to give a direct proof of uniqueness of solution of equation (3.24) as well as (3.33), respectively, let us clarify this point by means of the following arguments. We first note that in this subcase as well as in other subcases below the two curves $V\left(s ; B^{\prime}\right)$ and $V\left(s ; B^{\prime \prime}\right)$ do not intersect on the interval $\left(L, B^{\prime}\right]$ as solutions of the integro-differential equation (3.10) started at two different points $B^{\prime}$ and $B^{\prime \prime}$ according to the first condition in (2.7) whenever $K<B^{\prime}<$ $B^{\prime \prime}<H$. This can be shown by applying the arguments similar to [18, Remark 2.2] and [19, Theorem 4.1] or by verifying directly. Observe that we also have $0<V^{\prime}(K ; B)<1$ for all $B \in(K, H)$. Then, by using the fact that the function $V(s ; B)$ is convex on $[K, B]$ for each $B \in(K, H)$ fixed, we may conclude that if $\lim _{B \uparrow H} V^{\prime}(B-; B)>1$ then there exists a unique point $B_{*} \in(K, H)$ at which the curve $V\left(s ; B_{*}\right)$ hits the line $s-K$ smoothly implying that equation (3.24) has a unique solution on the interval $(K, H)$. Observe that if $\lim _{B \uparrow H} V^{\prime}(B-; B)<1$ then there is no solution on $(K, H)$ of $(3.24)$ as well as (3.33), respectively. The uniqueness of the roots of equations (3.34) and (3.35) below is verified straightforwardly.

Note that if, in addition, $\sigma=0$ holds with $r-\delta+\zeta>0$ and $\zeta=-\lambda \theta /(1-\theta)$, then we can put $C_{3}=0$ into (3.15) and (3.17) and ignore the second condition in (2.7) 
implying (3.23). Thus, solving the system $(3.20)+(3.22)+(3.24)$ with $C_{3}=0$, by using straightforward calculations we obtain that the solution of the system $(2.6)-(2.8)+(2.10)$ is given by:

$V\left(s ; B_{*}\right)=\left(B_{*}-K\right) \frac{\beta_{1}\left(s / B_{*}\right)^{\gamma_{1}}-\beta_{2}\left(s / B_{*}\right)^{\gamma_{2}}}{\beta_{1}-\beta_{2}}+\frac{\beta_{1} \beta_{2} B_{*}^{\alpha} F\left(B_{*}, H, K\right)}{\beta_{1}-\beta_{2}}\left[\left(\frac{s}{B_{*}}\right)^{\gamma_{1}}-\left(\frac{s}{B_{*}}\right)^{\gamma_{2}}\right]$

for all $0<L<s<B_{*}$, where $B_{*}$ is determined as the unique solution of the equation:

$$
F(B, H, K)=\frac{\beta_{1} \gamma_{1}-\beta_{2} \gamma_{2}}{\beta_{1}-\beta_{2}} \frac{B-K}{\beta_{1} \beta_{2} B^{\alpha}}-\frac{B}{\beta_{1} \beta_{2} B^{\alpha}}
$$

on the interval $(K, H)$ whenever it exists.

Observe that when $L=0$ and either $\sigma>0$, or $\sigma=0$ with $r-\delta+\zeta>0$ holds, we also ignore the second condition in (2.7) implying (3.23) and take into account the fact that if $\alpha=1 / \theta>0$ then $\beta_{3}<-\alpha<1-\alpha<\beta_{2}<0<\beta_{1}$ so that $\gamma_{3}<0<1<\gamma_{2}<\alpha<\gamma_{1}$ with $\gamma_{i}=\beta_{i}+\alpha$ for $i=1,2,3$. It thus follows that in (3.15) as well as in (3.17) we have $C_{3}=0$, since otherwise $V(s) \rightarrow \pm \infty$ as $s \downarrow 0$ that should be excluded by virtue of the fact that the value function (2.3) is bounded under $s \downarrow 0$. Therefore, solving the system $(3.20)+(3.22)+(3.24)$ with $C_{3}=0$ we obtain that the solution of the system (2.6)-(2.8) $+(2.10)$ is given by the same formulas as in (3.32)-(3.33).

3.5. Let us finally consider the subcase $\sigma=0$ and $\alpha=1 / \theta>1$ with $r-\delta+\zeta \leq 0$ and $\zeta=-\lambda \theta /(1-\theta)$. Observe that in this case we can put $C_{3}=0$ and omit the smooth-fit condition (2.10) implying (3.24). If, in addition, $r-\delta+\zeta<0$ holds, then solving the system $(3.20)+(3.22)-(3.23)$ with $C_{3}=0$, by using straightforward calculations we obtain that the solution of the system (2.6)-(2.8) is given by the same formula as in (3.6) with $\gamma_{i}=\beta_{i}+\alpha$ and $\beta_{i}$ for $i=1,2$ are given by (3.18), where $B_{*}$ is determined as the unique solution of the equation:

$$
F(B, H, K)=\frac{B-K}{\beta_{1} \beta_{2} B^{\alpha}} \frac{\beta_{1}(B / L)^{\gamma_{1}}-\beta_{2}(B / L)^{\gamma_{2}}}{(B / L)^{\gamma_{1}}-(B / L)^{\gamma_{2}}}
$$

on the interval $(K, H)$ whenever it exists.

Note that if, in addition, $r-\delta+\zeta=0$ holds, then we can put $C_{2}=C_{3}=0$ into (3.15) and (3.17) and ignore the second condition in (2.7) implying (3.23). Thus, solving the system $(3.20)+(3.22)$ with $C_{2}=C_{3}=0$, by using straightforward calculations we obtain 
that the solution of the system (2.6)-(2.8) is given by the same formula as in (3.8) with $\gamma_{1}=\beta_{1}+\alpha$ and $\beta_{1}$ is given by (3.19), where $B_{*}$ is determined as the unique solution of the equation:

$$
F(B, H, K)=\frac{B-K}{\beta_{1} B^{\alpha}}
$$

on the interval $(K, H)$ whenever it exists.

Observe that when $L=0$ we can take into account that if, in addition, $r-\delta+\zeta<0$ holds, then $\beta_{2}<-\alpha<1-\alpha<\beta_{1}<0$ so that $\gamma_{2}<0<1<\gamma_{1}$ with $\gamma_{i}=\beta_{i}+\alpha$, where $\beta_{i}$ for $i=1,2$ are given by (3.18). It follows that in (3.15) as well as in (3.17) we have $C_{2}=C_{3}=0$, since otherwise $V(s) \rightarrow \pm \infty$ as $s \downarrow 0$ that should be excluded by virtue of the fact that the function (2.3) is bounded under $s \downarrow 0$. Note that if, in addition, $r-\delta+\zeta=0$ holds, then $1-\alpha<\beta_{1}<0$ so that $\gamma_{1}>1$ with $\gamma_{1}=\beta_{1}+\alpha$, where $\beta_{1}$ is given by (3.19). Therefore, solving the system $(3.20)+(3.22)$ with $C_{2}=C_{3}=0$ we obtain that the solution of the system (2.6)-(2.8) is given by the same formulas as in (3.8) and (3.35), where if $r-\delta+\zeta<0$ then $\beta_{1}$ is given by (3.18), while if $r-\delta+\zeta=0$ then $\beta_{1}$ is given by (3.19).

\section{Main result and proof}

Taking into account the facts proved above, let us now formulate the main assertion of the paper.

Theorem 4.1. Let the process $S$ be given by (2.1)-(2.2). Then the value function of the optimal stopping problem (2.3) has the expression:

$$
V_{*}(s)= \begin{cases}V\left(s ; B_{*}\right), & \text { if } L<s<B_{*} \\ s-K, & \text { if } B_{*} \leq s<H \\ 0, & \text { if } 0<s \leq L \text { or } s \geq H\end{cases}
$$

and the optimal stopping time has the structure (2.4) whenever $B_{*}$ belongs to the interval $(K, H)$, where the function $V\left(s ; B_{*}\right)$ and the optimal stopping boundary $B_{*}$ are specified as follows: 
(i) if $\sigma>0$ and $\theta=0$ then $V\left(s ; B_{*}\right)$ is given by (3.6) with $B_{*}$ being the unique solution of (3.7) whenever it belongs to $(K, H)$;

(ii) if $\theta<0$ and either $\sigma>0$ or $\sigma=0$ then $V\left(s ; B_{*}\right)$ is given by (3.25) or (3.27) with $B_{*}$ being the unique solution of (3.26) or (3.28) whenever it belongs to $(K, H)$, respectively;

(iii) if $0<\theta<1$ and either $\sigma>0$, or $\sigma=0$ with $r-\delta-\lambda \theta /(1-\theta)>0$, then $V\left(s ; B_{*}\right)$ is given by (3.17) with $C_{i}=C_{i}\left(B_{*}, H, K, L\right)$ for $i=1,2,3$ defined by (3.29)-(3.31), or (3.32), with $B_{*}$ being the unique solution of (3.24) or (3.33) on $(K, H)$ whenever it exists, respectively;

(iv) if $\sigma=0$ and $0<\theta<1$ with either $r-\delta-\lambda \theta /(1-\theta)<0$ or $r-\delta-\lambda \theta /(1-\theta)=0$ then $V\left(s ; B_{*}\right)$ is given by (3.6) or (3.8) with $B_{*}$ being the unique solution of (3.34) or (3.35) on $(K, H)$ whenever it exists, respectively, where $\gamma_{i}$ are replaced by $\beta_{i}+\alpha$ and $\beta_{i}$ for $i=1,2$ are given by (3.18) in case $r-\delta-\lambda \theta /(1-\theta)<0$, and $\beta_{1}$ is given by (3.19) in case $r-\delta-\lambda \theta /(1-\theta)=0$.

Proof. In order to verify the assertions stated above, it remains to show that the function (4.1) coincides with the value function (2.3) and the stopping time $\tau_{*}$ from (2.4) with the boundary $B_{*}$ specified above is optimal. For this, let us denote by $V(s)$ the righthand side of the expression (4.1). In this case, by means of straightforward calculations and the assumptions above it follows that the function $V(s)$ solves the system (2.6)-(2.8), and the smooth-fit condition (2.10) is satisfied when either $\sigma>0$ or $r-\delta-\lambda \theta /(1-\theta)>0$ holds. In addition, we observe that when either $\sigma>0$ or $r-\delta-\lambda \theta /(1-\theta)<0$ holds, then $V(s)$ turns out to be a convex function on the set $\left[L, H^{\prime}\right]$ for each $H^{\prime} \in(K, H)$. Hence, by applying Itô-Tanaka-Meyer formula (see, e.g., [10, Chapter V, Theorem 5.52] or [21, Chapter IV, Theorem 70]) to $e^{-r t} V\left(S_{t}\right)$ we obtain:

$$
e^{-r t} V\left(S_{t}\right)=V(s)+\int_{0}^{t} e^{-r u}(\mathbb{L} V-r V)\left(S_{u}\right) I\left(S_{u} \neq B_{*}\right) d u+M_{t}
$$

for all $0 \leq t<\eta$, where $\eta=\inf \left\{t \geq 0 \mid S_{t} \notin(L, H)\right\}$ and the process $\left(M_{t}\right)_{0 \leq t<\eta}$ given by:

$$
\begin{aligned}
M_{t}= & \int_{0}^{t} e^{-r u} V^{\prime}\left(S_{u}\right) I\left(S_{u} \neq B_{*}\right) \sigma S_{u} d W_{u} \\
& +\int_{0}^{t} \int_{0}^{\infty} e^{-r u}\left(V\left(S_{u-} e^{\theta y}\right)-V\left(S_{u-}\right)\right)(\mu(d u, d y)-\nu(d u, d y))
\end{aligned}
$$


is a local martingale with respect to $P_{s}$ being a probability measure under which the process $S$ defined in (2.1)-(2.2) starts at $s \in(L, H)$. Remark that when $\sigma>0$, the smooth-fit condition (2.10) holds, so that there is no local time term in the formula (4.2). Observe that when $\sigma=0$ and $\theta \neq 0$ with $r-\delta-\lambda \theta /(1-\theta) \geq 0$, the function $V(s)$ is continuously differentiable on $(L, H)$. Thus, the expression (4.2) remains true by virtue of the classical change-of-variable formula (see, e.g., [21, Chapter II, Theorem 31]). Note that when $\sigma=0$ and $r-\delta-\lambda \theta /(1-\theta)=0$, the indicators in the formulas (4.2) and (4.3) can be set to one.

By using straightforward calculations and the arguments from the previous section, it can be verified that $(\mathbb{L} V-r V)(s) \leq 0$ for all $L<s<H$ and $s \neq B_{*}$. Moreover, by means of standard arguments it can be shown that the function $V\left(s ; B_{*}\right)$ is increasing on the interval $\left(L, B_{*}\right)$, and thus the property (2.9) also holds that together with (2.7)-(2.8) yields $V(s) \geq(s-K)^{+}$for all $L<s<H$. Observe that from (2.1) it is seen that when either $\sigma>0$ or $r-\delta-\lambda \theta /(1-\theta) \neq 0$, the time spent by the process $S$ at the point $B_{*}$ is of Lebesgue measure zero. Thus, in those cases, the indicators appearing in the integrals in (4.2)-(4.3) can be also ignored. Hence, from the expression (4.2) and the structure of the stopping time in (2.4) with $K<B_{*}<H$ it follows that the inequalities:

$$
e^{-r \tau}\left(S_{\tau}-K\right)^{+} \leq e^{-r \tau} V\left(S_{\tau}\right) \leq V(s)+M_{\tau}
$$

hold for any stopping time $\tau<\eta$ of the process $S$ started at $s \in(L, H)$.

Let $\left(\tau_{n}\right)_{n \in \mathbb{N}}$ be an arbitrary localizing sequence of stopping times for the process $\left(M_{t}\right)_{0 \leq t<\eta}$. Taking in (4.4) the expectation with respect to the measure $P_{s}$, by means of the optional sampling theorem (see, e.g., [11, Chapter I, Theorem 1.39]) we get:

$$
\begin{aligned}
& E_{s}\left[e^{-r\left(\tau \wedge \tau_{n}\right)}\left(S_{\tau \wedge \tau_{n}}-K\right)^{+}\right] \leq E_{s}\left[e^{-r\left(\tau \wedge \tau_{n}\right)} V\left(S_{\tau \wedge \tau_{n}}\right)\right] \\
& \leq V(s)+E_{s}\left[M_{\tau \wedge \tau_{n}}\right]=V(s)
\end{aligned}
$$

for all $L<s<H$. Hence, letting $n$ go to infinity and using Fatou's lemma, we obtain that for any stopping time $\tau<\eta$ the inequalities:

$$
E_{s}\left[e^{-r \tau}\left(S_{\tau}-K\right)^{+}\right] \leq E_{s}\left[e^{-r \tau} V\left(S_{\tau}\right)\right] \leq V(s)
$$

are satisfied for all $L<s<H$. 
By virtue of the fact that the function $V(s)$ together with the boundary $B_{*}$ satisfy the system (2.6)-(2.10) and taking into account the structure of $\tau_{*}$ in (2.4), from the expression (4.2) it follows that the equalities:

$$
e^{-r\left(\tau_{*} \wedge \tau_{n}\right)}\left(S_{\tau_{*} \wedge \tau_{n}}-K\right)^{+}=e^{-r\left(\tau_{*} \wedge \tau_{n}\right)} V\left(S_{\tau_{*} \wedge \tau_{n}}\right)=V(s)+M_{\tau_{*} \wedge \tau_{n}}
$$

hold for all $L<s<H$ and any localizing sequence $\left(\tau_{n}\right)_{n \in \mathbb{N}}$ of $\left(M_{t}\right)_{0 \leq t<\eta}$. Observe that by the structure of $\tau_{*}$ and $\eta$ as well as by the integrability of jumps of the process $S$, by using the independence of the processes $W$ and $J$ in the expression (2.1), it can be shown that the property:

$$
E_{s}\left[\sup _{t \geq 0} e^{-r\left(\tau_{*} \wedge t\right)} S_{\tau_{*} \wedge t}\right]<\infty
$$

holds for all $L<s<H$ and the variable $e^{-r \tau_{*}} S_{\tau_{*}}$ is equal to zero on the set $\left\{\tau_{*}=\infty\right\}$. Hence, letting $n$ go to infinity and using conditions (2.7)-(2.8), we can apply the Lebesgue dominated convergence theorem for (4.7) to obtain the equality:

$$
E_{s}\left[e^{-r \tau_{*}}\left(S_{\tau_{*}}-K\right)^{+}\right]=V(s)
$$

for all $L<s<H$, which together with (4.6) directly implies the desired assertion.

By using the facts proved in the previous section and by applying the same arguments as in the proof of Theorem 4.1, it is shown that the following assertion holds, which can be formally obtained as the limiting case of the main result under $L \downarrow 0$.

Corollary 4.2. Suppose that in the conditions of Theorem 4.1 we have $L=0$. Then the value function of the problem (2.3) takes the form:

$$
V_{*}(s)= \begin{cases}V\left(s ; B_{*}\right), & \text { if } 0<s<B_{*} \\ s-K, & \text { if } B_{*} \leq s<H \\ 0, & \text { if } \text { or } s \geq H\end{cases}
$$

and the optimal stopping time is given by (2.4) whenever $B_{*}$ belongs to the interval $(K, H)$, where $V\left(s ; B_{*}\right)$ and the optimal stopping boundary $B_{*}$ are specified as follows:

(i) if $\sigma>0$ and $\theta=0$ then $V\left(s ; B_{*}\right)$ is given by (3.8) with $B_{*}$ from (3.9) whenever it belongs to $(K, H)$, where $\gamma_{1}$ is given by (3.2); 
(ii) if $\theta<0$ then $V\left(s ; B_{*}\right)$ is given by (3.8) with $B_{*}$ from (3.9) whenever it belongs to $(K, H)$ and $\gamma_{1}$ replaced by $\beta_{1}+1 / \theta$, where $\beta_{1}$ is the largest root of the equation (3.16) in case $\sigma>0$, and $\beta_{1}$ is given by (3.18) in case $\sigma=0$;

(iii) if $0<\theta<1$ and either $\sigma>0$, or $\sigma=0$ with $r-\delta-\lambda \theta /(1-\theta)>0$, then $V\left(s ; B_{*}\right)$ is given by (3.32) with $B_{*}$ being the unique solution of (3.33) on $(K, H)$ whenever it exists, where $\gamma_{i}=\beta_{i}+1 / \theta$ and $\beta_{i}$ for $i=1,2$ are the largest roots of the equation (3.16) in case $\sigma>0$, and $\beta_{i}$ for $i=1,2$ are given by (3.18) in case $\sigma=0$;

(iv) if $\sigma=0$ and $0<\theta<1$ with either $r-\delta-\lambda \theta /(1-\theta)<0$ or $r-\delta-\lambda \theta /(1-\theta)=0$ then $V\left(s ; B_{*}\right)$ is given by (3.8) with $B_{*}$ being the unique solution of (3.35) on $(K, H)$ whenever it exists and $\gamma_{1}$ replaced by $\beta_{1}+1 / \theta$, where $\beta_{1}$ is given by (3.18) in case $r-\delta-\lambda \theta /(1-\theta)<0$, and $\beta_{1}$ is given by (3.19) in case $r-\delta-\lambda \theta /(1-\theta)=0$.

Let us now consider the question how changes in the volatility coefficient $\sigma$ affect the optimal exercise boundary $B_{*}$.

Remark 4.3. From the results of Theorem 4.1 it follows that the increase in $\sigma$ may expand the exercise (stopping) region $\left[B_{*}, \infty\right)$ of the perpetual American double barrier call option with irregular (non-convex) payoff function, since it may result into a faster exit of the process $S$ from $(L, H)$ after which the option become valueless. This stays in contrast with the general fact that increased volatility increases the values and expands the continuation regions of perpetual American options with convex exercise payoffs.

Let us finally consider the dependence of the solution on the lower barrier $L$.

Remark 4.4. Let us denote by $V_{*}(s ; L)$ the fair price of the perpetual American double barrier call option from (2.3) and by $B_{*}(L)$ the exercise boundary from (2.4), where we underline the dependence on $L \in(0, K)$. Then, by the structure of the payoff in $(2.3)$ it follows that $V_{*}(s ; L)$ decreases in $L$ on $(0, K)$. Hence, a simple comparison argument yields that $B_{*}(L)$ also decreases in $L$ on $(0, K)$. The intuition behind these properties is that the holder should exercise an option with a higher floor $L$ earlier than an option with a lower one. 


\section{Conclusion}

We have considered the perpetual American double barrier call option problem in a jumpdiffusion model with infinite time horizon. The related irregular optimal stopping problem has been reduced to a nontrivial free-boundary problem which has been solved under different relationships on the parameters of the model. The behavior of the solution under the changing lower barrier has been also studied. Let us finally make some concluding remarks concerning the analytic properties of the obtained solution of the free-boundary problem under several relationships on the parameters of the model.

Remark 5.1. Observe that when $\sigma=0$ and $0<\theta<1$ with $r-\delta-\lambda \theta /(1-\theta) \leq 0$ we have $V_{*}^{\prime}\left(B_{*}-\right)<1$ and thus the smooth-fit condition (2.10) fails to hold (see Figure 1). This property can be explained by the fact that in this case, leaving the continuation region $\left(L, B_{*}\right)$ the process $S$ can pass through the boundary $B_{*}<H$ only by jumping. Such an effect was earlier observed and explained in [18, Section 2] and [19] by solving other optimal stopping problems for jump processes.

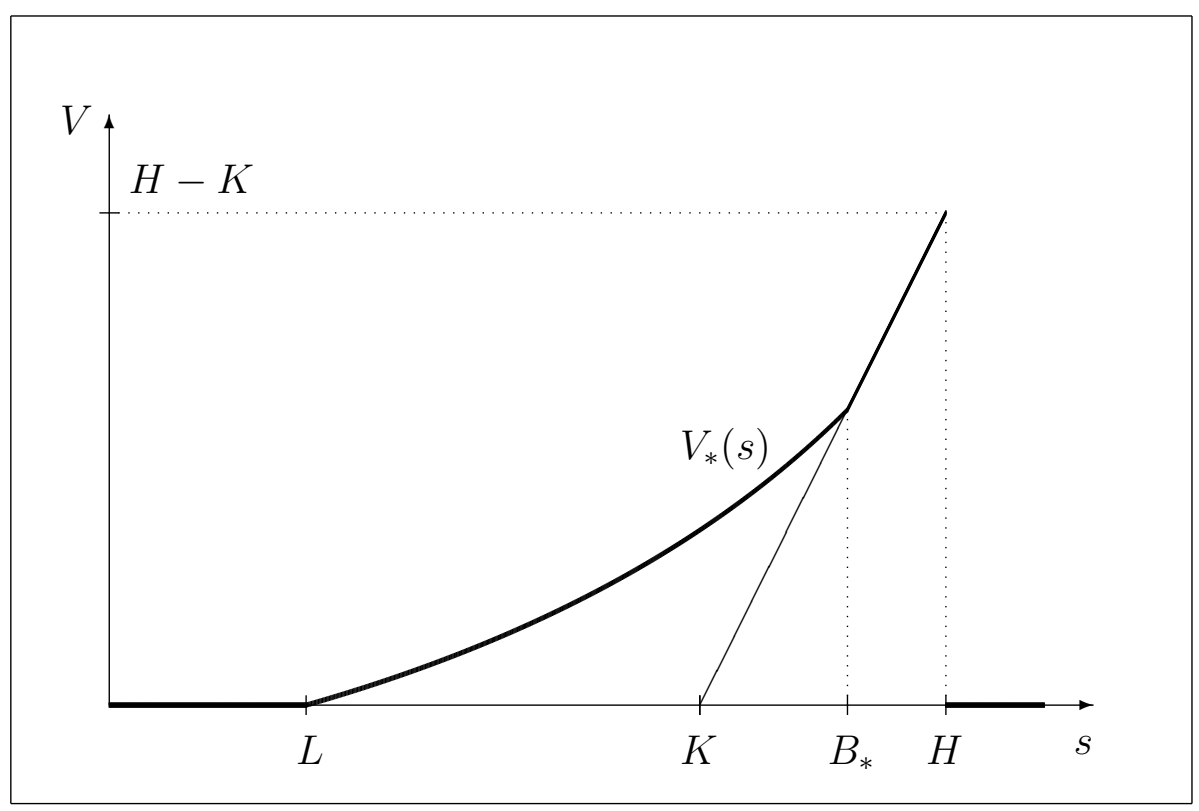

Figure 1. A computer drawing of the value function $V_{*}(s)$ and the boundaries $L$ and $B_{*}$ in the case of Remark 5.1. 
Remark 5.2. Note that when either $\sigma>0$ or $r-\delta-\lambda \theta /(1-\theta)>0$, the solution $B_{*}$ of equations (3.5) and (3.24) may coincide with the given upper barrier $H$. This means that $V_{*}^{\prime}(H-)=1$ may hold, which is equivalent to the smooth-fit condition (2.10) (see Figure 2), but at the same time, the boundary $H$ is not optimal. This property can be explained by the discontinuity of the payoff function in (2.3) at the point $H$ in these cases.

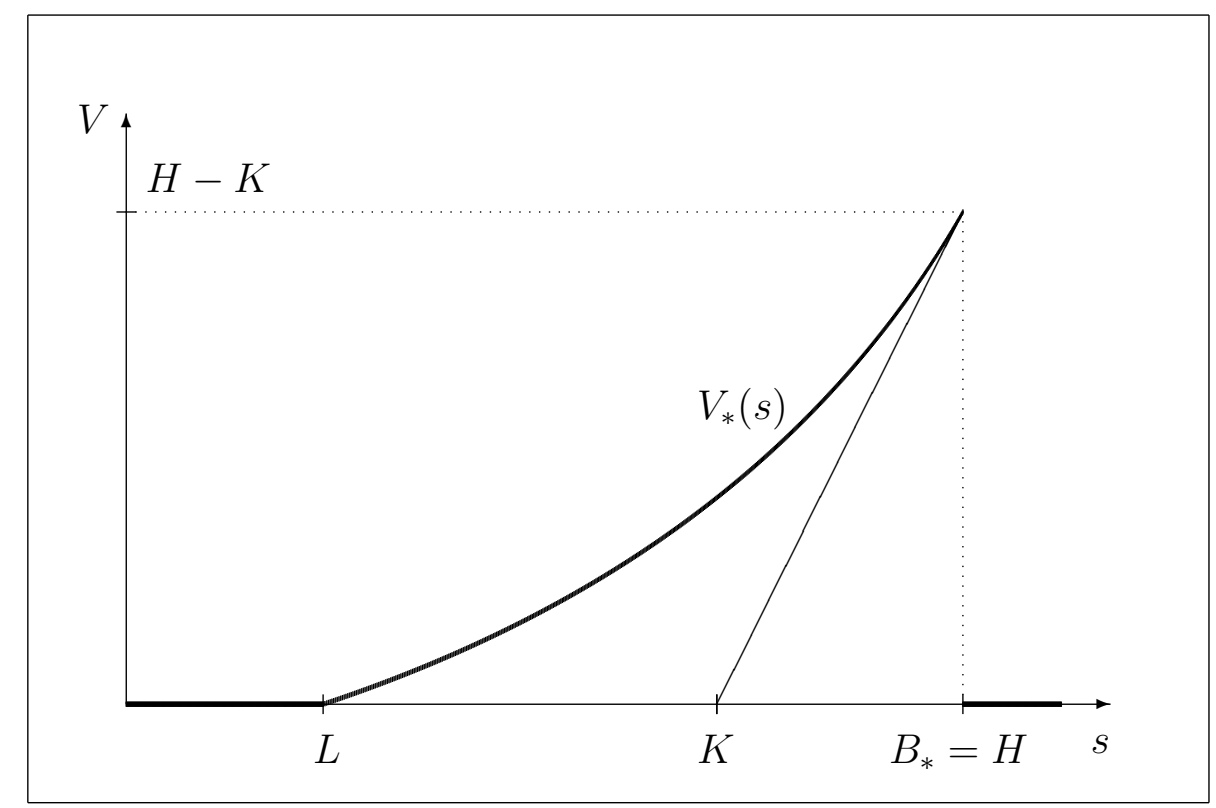

Figure 2. A computer drawing of the value function $V_{*}(s)$ and the boundaries $L$ and $B_{*}$ in the case of Remark 5.2.

Remark 5.3. Observe that when $\sigma=0$ and $\theta<0$ we have $V_{*}(L+)>0$ and thus the second condition in (2.7) fails to hold (see Figure 3). This property can be explained by the fact that $r-\delta-\lambda \theta /(1-\theta)>0$ under $\theta<0$, so that leaving the continuation region $\left(L, B_{*}\right)$ the process $S$ can pass through the fixed boundary $L$ only by jumping. Such an effect was earlier observed and explained in [18, Section 3] (see also [1] and [6]).

The continuity of the value function in optimal stopping problems with discontinuous rewards was studied in [2]-[3] and [5]. According to the results in [1] and [6] we may conclude that the properties described in Remarks 5.1-5.3 appear because of finite intensity of jumps and exponential distribution of jump sizes of the compound Poisson process $J$.

Remark 5.4. Note that when $\sigma=0$ with $0<\theta<1$ and $r-\delta-\lambda \theta /(1-\theta) \geq 0$ the 
value function $V_{*}(s)$ and the stopping boundary $B_{*}$ do not depend on the lower barrier $L$. This property can be explained by the fact that in this case the process $S$ is strictly increasing and thus it can never pass through the fixed boundary $L$ after being started at $s \in(L, H)$.

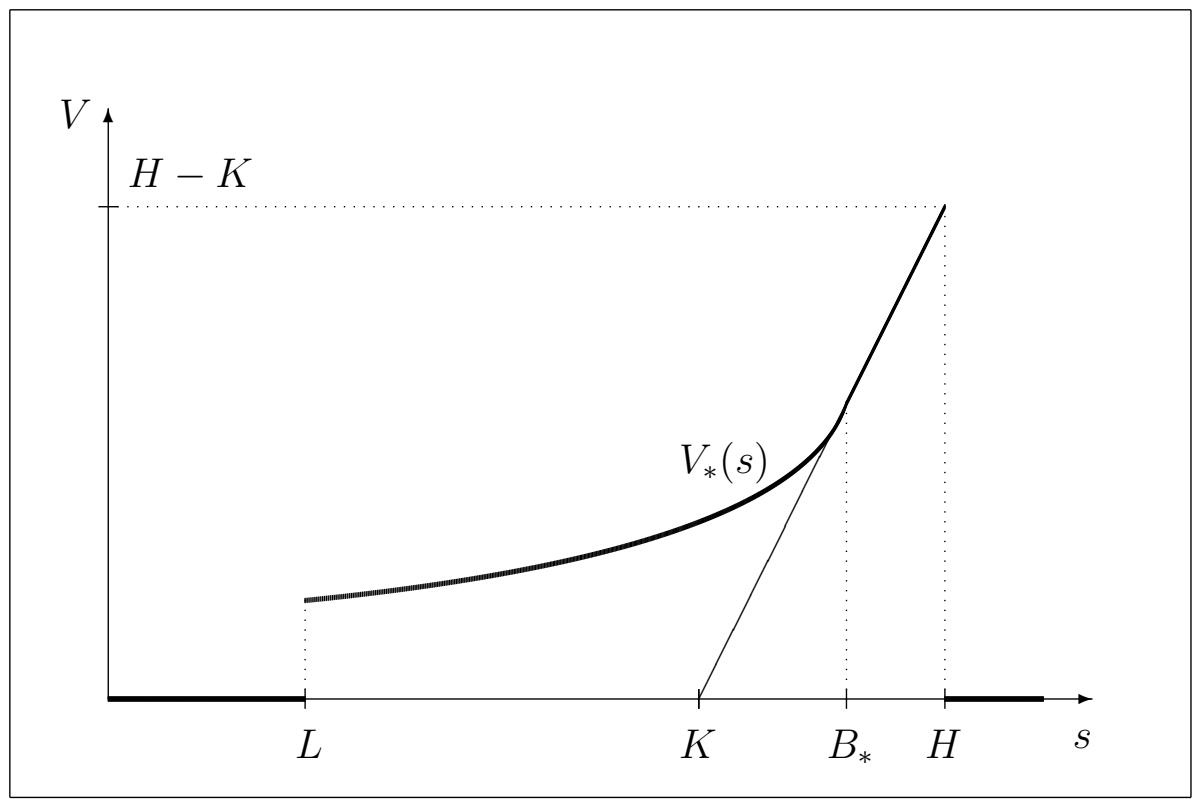

Figure 3. A computer drawing of the value function $V_{*}(s)$ and the boundaries $L$ and $B_{*}$ in the case of Remark 5.3.

Acknowledgments. The results of the paper were presented at the Symposium on Optimal Stopping with Applications held at the University of Manchester in January 2006. The author is grateful to the organizers and participants for their interest and useful comments. The author is indebted for both anonymous referees for their valuable suggestions, which helped to revise the paper.

\section{References}

[1] Alili, L. and Kyprianou, A. E. (2005). Some remarks on first passage of Lévy processes, the American put and pasting principles. Annals of Applied Probability 15(3) (2062-2080).

[2] Bassan, B. and Ceci, C. (2000). Optimal stopping problems with discontinuous 
reward: regularity of the value function and viscosity solutions. Stochastics and Stochastics Reports 72(1-2) (55-77).

[3] Bassan, B. and Ceci, C. (2002). Regularity of the value function and viscosity solutions in optimal stopping problems for general Markov processes. Stochastics and Stochastics Reports 74(3-4) (633-649).

[4] Broadie, M. and Detemple, J. (1995). American capped call options on dividend-paying assets. Review of Financial Studies 8(1) (161-191).

[5] Ceci, C. and Bassan, B. (2004). Mixed optimal stopping and stochastic control problems with semicontinuous final reward for diffusion processes. Stochastics and Stochastics Reports 76(4) (323-337).

[6] Cont, R. and Voltchkova, E. (2005). Integro-differential equations for option prices in exponential Lévy models. Finance and Stochastics 9 (299-325).

[7] Dynkin, E. B. (1963). The optimum choice of the instant for stopping a Markov process. Soviet Math. Dokl. 4 (627-629).

[8] Gapeev, P. V. and Kühn, C. (2005). Perpetual convertible bonds in jumpdiffusion models. Statistics and Decisions 23 (15-31).

[9] Grigelionis, B. I. and Shiryaev, A. N. (1966). On Stefan's problem and optimal stopping rules for Markov processes. Theory Probab. Appl. 11 (541-558).

[10] JaCOD, J. (1979). Calcul Stochastique et Problèmes de Martingales. Lecture Notes in Mathematics, Berlin.

[11] Jacod, J. and ShiRyaev, A. N. (1987). Limit Theorems for Stochastic Processes. Springer, Berlin.

[12] Kallsen, J. and KüHn, C. (2004). Pricing derivatives of American and game type in incomplete markets. Finance and Stochastics 8 (261-284).

[13] Karatzas, I. and Wang, H. (2000). Barrier options of American type. Applied Mathematics and Optimization 42 (259-279). 
[14] Kou, S. G. (2002). A jump diffusion model for option pricing. Management Science 48 (1086-1101).

[15] Kou, S. G. and Wang, H. (2004). Option pricing under a double exponential jump diffusion model. Management Science 50 (1178-1192).

[16] Mordecki, E. (1999). Optimal stopping for a diffusion with jumps. Finance and Stochastics $\mathbf{3}$ (227-236).

[17] Mordecki, E. (2002). Optimal stopping and perpetual options for Lévy processes. Finance and Stochastics 6 (473-493).

[18] Peskir, G. and Shiryaev, A. N. (2000). Sequential testing problems for Poisson processes. Annals of Statistics 28 (837-859).

[19] Peskir, G. and Shiryaev, A. N. (2002). Solving the Poisson disorder problem. Advances in Finance and Stochastics. Essays in Honour of Dieter Sondermann. Sandmann, K. and Schönbucher, P. eds. Springer (295-312).

[20] Peskir, G. and Shiryaev, A. N. (2006). Optimal Stopping and Free-Boundary Problems. Bikkhäuser, Basel.

[21] Protter, Ph. E. (2004). Stochastic Integration and Differential Equations. (Second Edition) Springer, Berlin.

[22] Shepp, L. A., Shiryaev A. N. and Sulem, A. (2002). A barrier version of the Russian option. Advances in Finance and Stochastics. Essays in Honour of Dieter Sondermann. Sandmann, K. and Schönbucher, P. eds. Springer (271-284).

[23] Shiryaev, A. N., Kabanov, Y. M., Kramkov, D. O. and MelNIKOV, A. V. (1994). On the pricing of options of European and American types, II. Continuous time. Theory of Probability and Applications 39(1) (61-102).

[24] Shiryaev, A. N. (1978). Optimal Stopping Rules. Springer, Berlin.

[25] Shiryaev, A. N. (1999). Essentials of Stochastic Finance. World Scientific, Singapore. 